\title{
miR-148b reverses cisplatin-resistance in non-small cell cancer cells via negatively regulating DNA (cytosine-5)-methyltransferase 1(DNMT1) expression
}

\author{
Chengguang Sui, Fandong Meng, Yan Li and Youhong Jiang
}

\begin{abstract}
Background: The emergence of drug resistance in cancer patients limits the success rate of clinical chemotherapy. MicroRNAs (miRNAs) may play a role in chemoresistance and may be involved in modulating of some drug resistance-related pathways in cancer cells. In this study, the involvement of microRNA-148b (miR-148b) and its roles in the development of chemoresistance in lung cancer are determined.

Methods: This study was performed in two lung cancer cell lines (A549 and SPC-A1). The levels of miR-148b and DNMT1 mRNA expression were determined by using Quantitative Real-Time PCR. Proteins of DNMTs are represented by western blot assay. Cell viability was assessed by MTT assay. Cell apoptosis was evaluated using flow cytometry.

Results: The data showed a down-regulated of miR-148b expression and evaluated methyltransferases (DNMTs) expression in cisplatin-resisted human non-small cell lung cancer (NSCLC) cell line-A549/DDP and SPC-A1/DDP compared with their parental A549 and SPC-A1 cell line. In transfection experiments, miR-148b mimics reduced the DNMT1 expression, as well as enhanced the sensitivity of cells to cisplatin and cisplatin-induced apoptosis in A549/DDP or SPC-A1/DDP cells. While miR-148b inhibitor increased DNMT1 expression, as well as attenuated the sensitivity of cells to cisplatin in A549 and SPC-A1 cells. miR-148b was showed to exert negative effect on DNMT1 expression by targeting its $3^{\prime} U T R$ in A549/DDP and A549 cells. Importantly, silenced DNMT1 increases cisplatin sensitivity of A549/DDP cells and over-expressed DNMT1 reverses pro-apoptosis effect of miR-148b mimic.
\end{abstract}

Conclusions: miR-148b reverses cisplatin-resistance in non-small cell cancer cells via negatively regulating DNMT1 expression.

Keywords: DNA (cytosine-5)-methyltransferase 1(DNMT1), Cisplatin resistance, MicroRNA-148b, A549 cell, SPC-A1 cell

\section{Background}

Lung cancer is regarded as the leading cause of cancer related deaths worldwide [1]. Among this, non-small cell lung cancer (NSCLC), which distinguished to small-cell lung cancer (SCLC) from pathological and histological characteristics, represents approximately $85 \%$ [2] and its five-year survival is only $15 \%$ [3]. Chemotherapy is well known as the main method to treat lung cancer in earlier stages of treatment, especially as adjuvant chemotherapy

\footnotetext{
*Correspondence: Chengguang_S@163.com

Molecular Oncology Department of Cancer Research institution, The First Hospital of China Medical University, 155 Nanjing North Street, He-ping District of Shenyang City, Liaoning Province 110001, China
}

after surgery. Although advances in cisplatin-based chemotherapy have resulted in improving the rate of survival, their therapeutic efficacy was limited for the development of cisplatin resistance. Therefore, a good understanding of the molecular mechanisms underlying cisplatin resistance development is urgently needed.

microRNAs are small non-coding RNA molecules consisting of 20-24 nucleotides and function as the suppressor for gene expression by interacting with the $3^{\prime}$ untranslated regions ( $3^{\prime}$ UTRs) of target mRNAs at 5-7 nucleotides. These interactions may lead to either obstruction of translation or degradation of the targeted mRNAs [4]. Dysregulation of miRNAs in cells may 
result in alterations in cellular differentiation, proliferation, apoptosis and metastasis processes that are important in the development of cancer [5]. Recently, accumulated studies have shown that miRNAs may play a role in chemoresistance of cancer treatment and may be involved in the modulation of some drug resistance-related pathways in some cancer cells.

Although few studied focus on the involvement of microRNA-148b (miR-148b) in drug resistance-related miRNAs, it has been described to be down-regulated in several types of cancers including pancreatic cancer $[6,7]$ colorectal cancer [8], gastric cancer [9], and basal-line breast cancer [10]. Furthermore, recent report indicates that miR-148b acts as a tumor suppressor by targeting specific oncogenes in NSCLC cells [11]. Importantly, miR-148a, which is together with miR-148b belonging to miR-148/152 family, plays an important role in improving response to chemotherapy in sensitive and resistant cancers. DNA methyltransferases (DNMTs), functioned as an important regulator for epigenetic processes of chemotherapy [12], have been proved to be regulated by miR-148b [7]. Thus, we hypothesized that miR-148b might be involved in chemotherapy resistance of NSCLC.

In this study, we focused on the effect of altered miR148b expression on cisplatin resistance of A549/DDP and SPC-A1/DPP cells. We also investigated the possible targets for miR-148b. This study will help to better understand the biological activities of miR-148b in NSCLC treatment.

\section{Methods}

\section{Cells culture and transfection}

Human lung adenocarcinoma cell line (A549 and SPC-A1) and their cisplatin-resistant variant A549/DDP and SPC-A1/DDP (purchased from the Academy of Military Medical Science, Beijing, P.R. China.) were cultured in RPMI-1640 medium (Invitrogen, Carlsbad, CA, USA) supplemented with $10 \%$ fetal calf serum (Gibco), penicillin $(100 \mu \mathrm{g} / \mathrm{mL})$, and streptomycin $(100 \mathrm{mg} / \mathrm{mL})$ in a humidified atmosphere containing $5 \% \mathrm{CO} 2$ at $37^{\circ} \mathrm{C}$. In order to maintain cisplatin-resistant phenotype, A549/DDP and SPC-A1/DDP cells were maintained in the medium additionally contained $2 \mu \mathrm{g} / \mathrm{mL}$ cisplatin (DDP).

The cells were seeded in 6-well plates at $1 \times 10^{5}$ cells/ well followed by cultured for 24 hours and then transfected with $25 \mathrm{nmol}$ of miR-148b mimics and negative control mimics (NC), miR-148b inhibitors (anti-miR-148b) and negative control inhibitors (anti-NC) (RIBO Bio, Guangzhou, P.R. China) using Lipofectamine 2000 (Invitrogen), according to the manufacturer's protocol. The effect of mimics or antagomirs of miR-148b was examined in triplicate at $24 \mathrm{~h}$ post-transfection. DNMT1 gene was knocked down using DNMT1 interfering small RNA (siRNA), which was obtained from Generay (Generay,
Shanghai, China) and transfected into the A549/DDP cells by Lipofectamine 2000 .

\section{Quantitative REAL-TIME PCR}

Total RNA was extracted from the cells with the Trizol Reagent (Invitrogen, Carlsbad, CA, USA). Quantitative Real-Time PCR (Q-RT-PCR) assays was to quantify the mature miR-148a and DNMT1 mRNA using fluorescent nucleic acid dye. Each sample $(1 \mu \mathrm{g})$ was reversetranscribed into cDNA by using the RevertAidTM First Strand cDNA Synthesis Kit (Fermentas). Real-time PCR was performed in the Applied Biosystems 7500 Real-time PCR system using SYBR Premix ExTagTM (Takara) according to the manufacturer's protocols. All reactions were run in triplicate. The threshold cycle $(\mathrm{CT})$ is defined as the fractional cycle number at which the fluorescence passes the fixed threshold. The miR-148b expression levels were normalized to U6 RNA. And the DNMT1 mRNA was normalized to $\beta$-actin mRNA. The relative expression was calculated using the comparative CT method.

\section{3-(4,5-dimethylthiazol-2-yl)-2,5- diphenyltetrazolium bromide (MTT) Assay}

The cells separately were seeded in 96-well plates at a density of $4 \times 103$ cells/well in culture medium. After their adherence to the culture dish, cells were incubated with mimic or inhibitor for miR148b for 24 hours. And then A549/DDP cells were treated with cisplatin at a series of concentrations $(10,20,40,80,160$ and $320 \mu \mathrm{g} / \mathrm{mL})$, while A549 cells were treated with cisplatin at 2, 4, 8, 16, 32 and $64 \mu \mathrm{g} / \mathrm{mL}$. Next, cells were cultured for an additional 48 hours and cell survival was measured by MTT assay according to the manufacturer's instructions. The resulting absorbance at $490 \mathrm{~nm}$ was measured on a spectrophotometer. The concentration at which drug produced 50\% inhibition of growth (IC50) was estimated by the relative survival curve.

\section{Western blot}

The cells were lyzed in RIPA buffer in the presence of proteinase inhibitor (Sigma-Aldrich, St. Louis, MO, USA). Equal amounts of protein was separated on 10\% sodium dedecyl sulfate polyacrylamide gel electrophoresis (SDSPAGE) and transferred to PVDF membrane (Millipore Corp, Billerica, MA, USA). Membranes were blocked with $10 \%$ skimmed milk followed by incubation with the antibodies (1:500): anti-DNMT1(sc-10222; Santa Cruz, CA, USA), anti-DNMT3a (sc-20703; Santa Cruz, CA, USA), anti-DNMT3b ( sc-10236; Santa Cruz, CA, USA) and anti- $\beta$-Actin (\#12620; Cell Signaling Technology, MA, USA). After washed extensively with $0.1 \%$ in phosphatebuffered saline (PBS), the membranes were incubated with secondary antibodies (anti-rabbit, 1:1000) conjugated with horseradish peroxidase. The reaction was detected 
with enhanced chemiluminescence. Protein levels were normalized to $\beta$-Actin.

\section{Flow cytometry-based apoptosis}

The A549/DDP cells were seeded in 96-well plates at a density of $4 \times 103$ cells/well in culture medium and transfected. Twenty-four hours later, $10 \mu \mathrm{g} / \mathrm{mL}$ cisplatin was added to the medium and the cells were incubated for 48 hours. After incubation, the cells were harvested, stained with $5 \mu \mathrm{L}$ annexin V-flourescein isothiocyanate (FITC) and $10 \mu \mathrm{L}$ propidium iodide (PI) $(20 \mu \mathrm{g} / \mathrm{mL})$. The mixture was incubated at room temperature in the dark for 15 minutes and analyzed by fluorescence-activated cell sorting (FACS).

\section{Bioinformatics analysis of miR-148b target in DNMT1}

Based on bioinformatics analysis, we predicted that hsamiR-148b can bind with the 3'UTR region of NADM1 by using four common websites (Target Scan: http:// www.targetscan.org/, miRanda: http://www.microrna.org/,
Microcosm: http://www.ebi.ac.uk/enright-srv/microcosm/ cgi-bin/targets/v5/genome.pl, and PITA: http://genie.weizmann.ac.il/) (Figure 1A).

\section{Plasmid construction and Luciferase reporter assays}

For DNMT1 3'UTR reporter assay, cells were placed in 24-well plates $(1 \times 105$ cells per well $)$ and then cotransfected with pGL3-DROSHA 3'UTR-T or pGL3-DROSHA 3'UTR-C and pRL-SV40 (50:1). The mimics and inhibitors of hsa-miR-148b and their negative controls (RIBO Bio, Guangzhou, P.R. China) were cotransfected with the reporter plasmids at a final concentration of $20 \mathrm{nmol} / \mu \mathrm{l}$. 48 hours after transfection in A549/DDP and A549 cells, luciferase activity in lysates was measured with a DualLuciferase Reporter Assay System (Promega, WI, USA) and normalized against the activity of the pRL-SV40. The assays were conducted followed by the manufacture's suggestions. Independent triplicate experiments were performed for each plasmid construct.
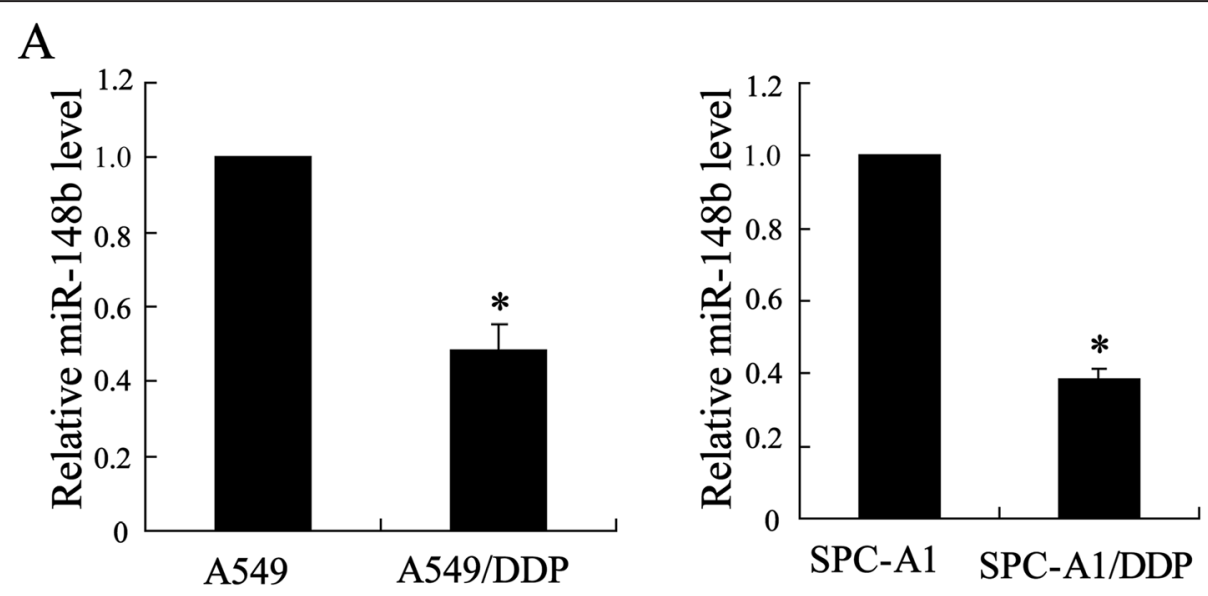

B

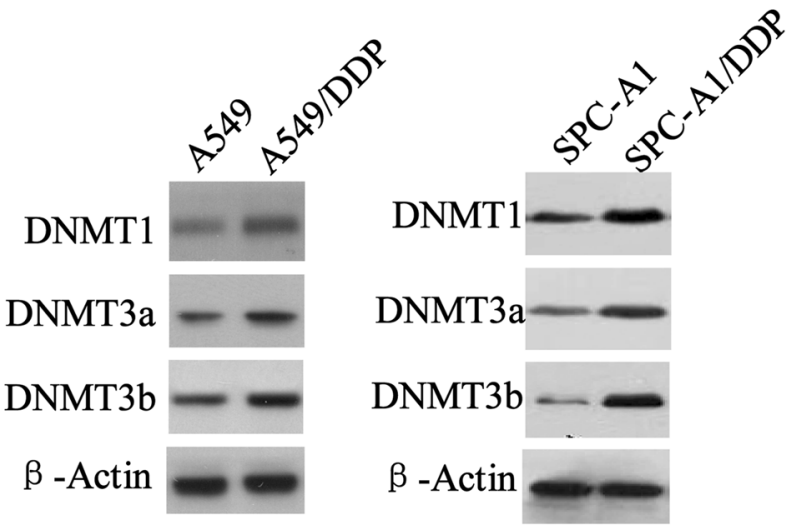

Figure 1 Expression of miR-148b and DNMTs in two cell lines of lung cancer. Cells were lyzed to detect level of (A) miR-148b using Q-RT-PCR and (B) NDMT1, NDMT3a and NDMT3b using western blot assay in A549/DDP, SPC-A1/DDP and their respective parental cells. Data were represented as mean \pm SD. ${ }^{*} P<0.05$ compared with A549. 


\section{Statistical analysis}

All experiments were run in triplicate. All statistical analysis were performed using SPSS 13.0. Data were expressed as means \pm standard deviation (SD). The difference between the groups was analyzed using Student's t test when only two groups were compared or one-way analysis of variance (ANOVA) when more than two groups were compared. Values of $P<0.05$ indicate statistical significance.

\section{Results}

Differential expression of miR-148b and DNMTs in both A549/DPP and SPC-A1/DPP cells compared with their parental cell lines

We detected the expression levels of miR-148b and DNMTs protein in A549/DPP and SPC-A1/DPP as well as their parental cell lines using Q-RT-PCR and Western blot, respectively. The results showed that miR-148b had
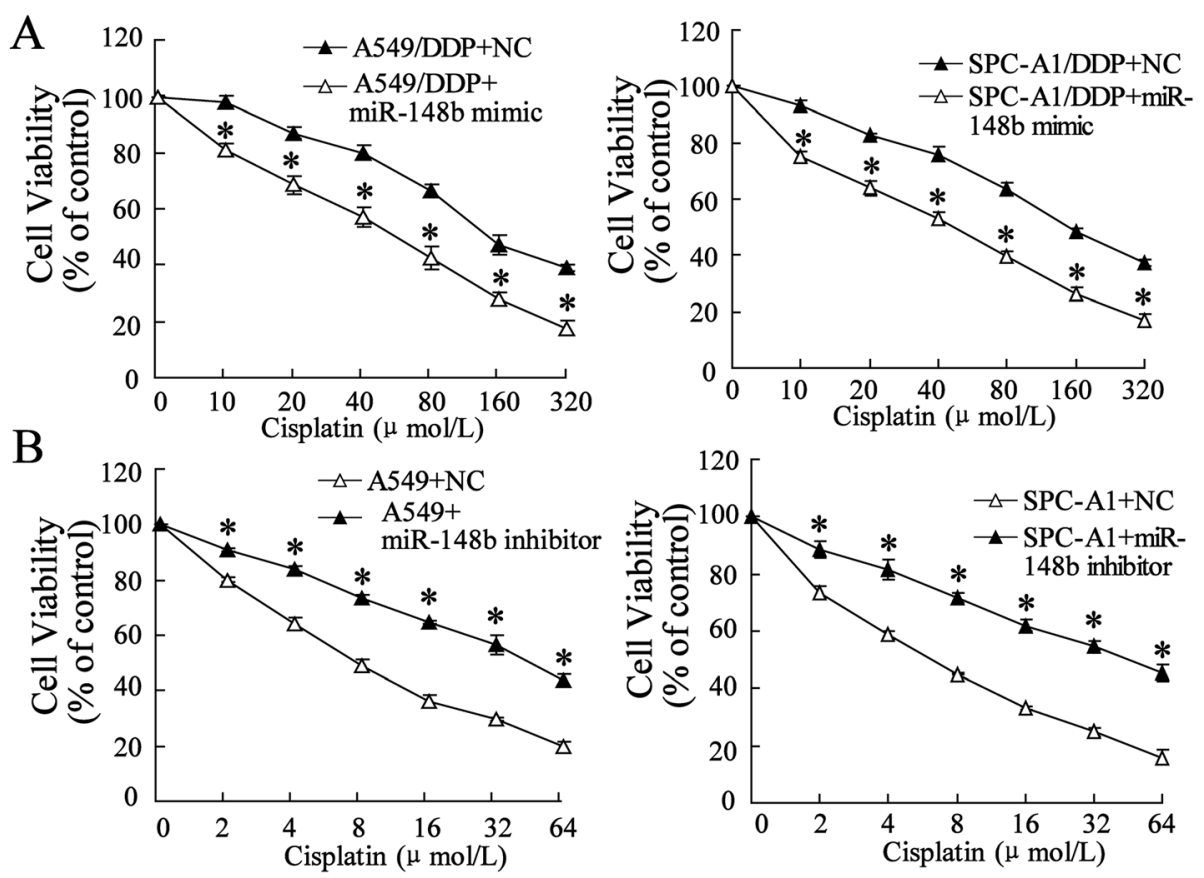

$\mathrm{C}$
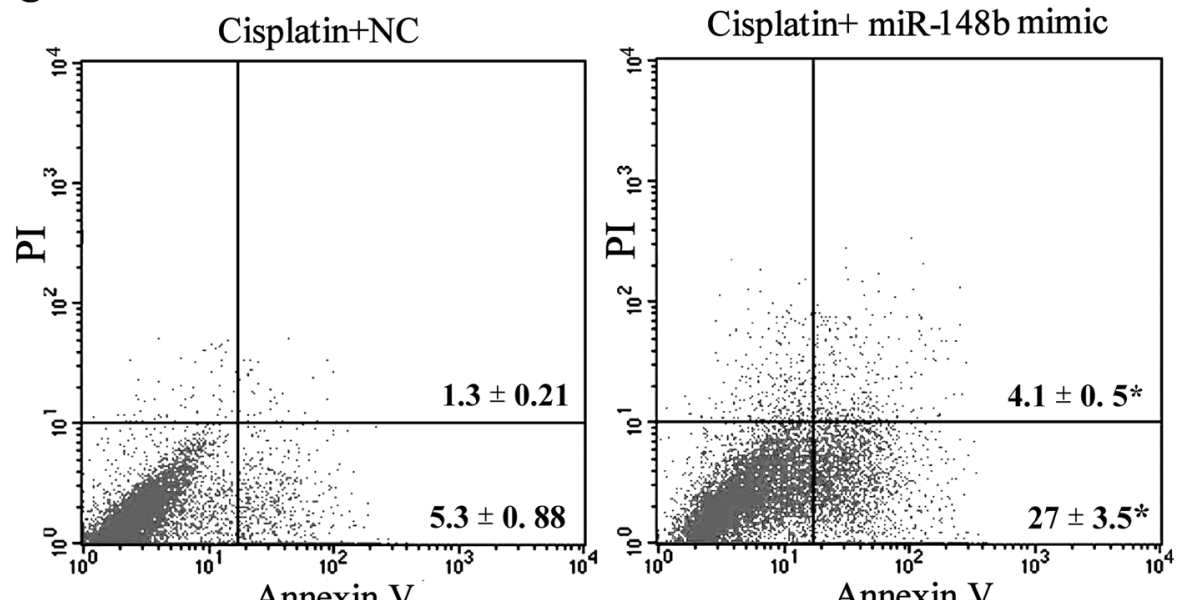

Figure 2 miR-148a modified the cisplatin sensitivity of A549/DDP and SPC-A1/DDP cells. (A) After A549/DDP and SPC-A1/DDP cells transfected by miR-148b mimic or NC for $24 \mathrm{~h}$, the cells were incubated with various doses of cisplatin $(10,20,40,80,160,320 \mu \mathrm{mol} / \mathrm{L})$ for $48 \mathrm{~h}$, and then cells viability was assessed by MTT assay. In A549/DDP cell, IC50miR-148b mimic is $55.36 \mu \mathrm{mol} / \mathrm{L}$, IC50NC is $173.27 \mu \mathrm{mol} / \mathrm{L}$; in SPC-A1/DDP cell, IC50miR-148b mimic is $44.25 \mu \mathrm{mol} / \mathrm{L}$, IC50NC is $156.32 \mu \mathrm{mol} / \mathrm{L}$. (B) After A549 and SPC-A1 cells transfected by miR-148b inhibitor or NC for $24 \mathrm{~h}$, the cells were incubated with various doses of cisplatin $(2,4,8,16,32 \mu \mathrm{mol} / \mathrm{L})$ for $48 \mathrm{~h}$, and then cells viability was assessed by MTT assay. In A549 cell, IC50miR-148b inhibitor is $45.06 \mu \mathrm{mol} / \mathrm{L}$, IC50NC is $9.21 \mu \mathrm{mol} / \mathrm{L}$; in SPC-A1 cell, IC50miR-148b inhibitor is $44.62 \mu \mathrm{mol} / \mathrm{L}$, IC50NC is $6.97 \mu \mathrm{mol} / \mathrm{L}$. (C) After A549/DDP cells treated by NC or miR-148b mimic for $24 \mathrm{~h}$, and incubated with $10 \mu \mathrm{mol} / \mathrm{L}$ cisplatin for $48 \mathrm{~h}$, cells apoptosis was evaluated using flow cytometry. Data were represented as mean \pm SD. ${ }^{*} P<0.05$ compared with cells treated with the same concentration of ciaplatin accordingly. 
an average of 2-fold lower expression level in A549/DPP cells and an average of 2.5 -fold lower expression level in SPC-A1/DPP cells than that in A549 cells and SPC-A1 cell, respectively $(P<0.05$, Figure $2 \mathrm{~A})$. On the contrary, the expression of DNMT1, DNMT3a and DNMT3b was upregulated in A549/DPP and SPC-A1/DPP cell lines (Figure 2B).

\section{Upregulation of miR-148b increased sensitivity of the A549/DDP and SPC-A1/DPP cells to cisplatin}

To investigate whether miR-148b could modulate the sensitivity of the A549/DPP and SPC-A1/DPP as well as their parental cell lines to cisplatin, we transfected cisplatin-resistant cells with mimics of miR-148b and their parental cells with inhibitors of miR-148b, respectively, and then the cells were treated with a series of concentrations of cisplatin. We got the data that the mimics of miR-148b increased the sensitivity to cisplatin significantly by 1.5 -fold in A549/DDP and SPC-A1/DPP cells $(P<0.05$, respectively; Figure 3A), however, sensitivities of A549 and SPC-A1 cells transfected with miR-148b inhibitor to cisplatin were decreased compared with that treated with $\mathrm{NC}(P<0.05$, respectively; Figure $3 \mathrm{~B})$. To further assess the role of miR-148b in regulating growth of A549/DDP and SPC-A1/DPP cells exposed to cisplatin, cells apoptosis rate that had been transfected with miR148b mimic were analyzed using flow cytometry assay. As shown in Figure 3C, compared with NC, miR-148b mimic promoted cisplatin-induced apoptosis of A549/DDP and SPC-A1/DPP cells $(P<0.05$, respectively).

\section{MiR-148b regulates expression of DNMT1}

To determine whether miR-148b is involved in regulation the expression of DNMTs, A549/DDP and A549 cells were transfected with the mimics or inhibitors of miR-
$148 \mathrm{~b}$ respectively, and the expression levels of DNMTs were determined by Western blot. Figure 4A showed that transfected mimics of miR-148b resulted in downregulating expression of DNMT1 in A549/DDP cells, but mimics of miR-148b exerted no effect on expression of DNMT3a and DNMT3b. In addition, expression of DNMT1 in A549 transfected with miR-148b inhibitor was upregulated, however, expressions of DNMT3a and DNMT3b were not changed (Figure 4B).

\section{Targeting site of miR-148 in the DNMT1 3'UTR}

DNMT1 was regarded as a potential target gene of miR148b using TargetScan Release 5.2 in which we found a binding site for miR-148b in the 3'UTR of DNMT1 mRNA (Figure 1A). To confirm DNMT1 as a real miR-148b target, the entire 3'UTR of DNMT1 was inserted downstream of the luciferase gene and assayed in A549/DDP and A549 cells. As shown in Figure 1B, cotransfection of miR-148b mimics with the DNMT1 3'UTR reporter resulted in a decrease (40\%) in luciferase activity and a highly significant decline in mRNA of DNMT1 in A549/DDP cells. Additionally, Figure $1 \mathrm{C}$ showed increases (2.3 fold) in luciferase activity and mRNA of DNMT1 in A549 transfected with miR-148b inhibitor and the DNMT1 3'UTR reporter. These data suggested a might involvement of DNMT1 in modulating the cisplatin sensitivity by miR-148b in the A549/DPP and A549 cells.

\section{DNMT1 siRNA increases cisplatin sensitivity of A549/DDP and SPC-A1/DPP cells}

To demonstrate the effect of DNMT1 on cisplatin sensitivity of lung cancer cells, the DNMT1 siRNA or siRNA negative control was transfected into the A549/DDP and SPC-A1/DPP cells to observe cell viability. We first measured the expression of DNMT1 using western blot and
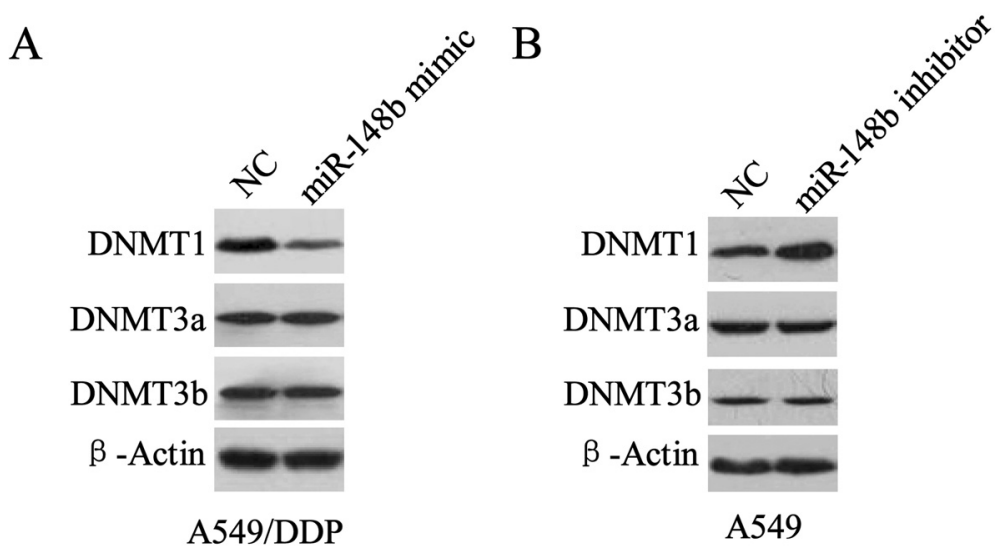

Figure 3 miR-148a modified expression of DNMTs. A549/DDP and A-549 cells were separately transfected by NC or miR-148b mimic or miR-148b ainhibitor for $24 \mathrm{~h}$. Expressions of DNMT1, DNMT3a and DNMT3b in cells were determined using western blot assay in (A) A549/DDP cells and (B) A549 cells. 


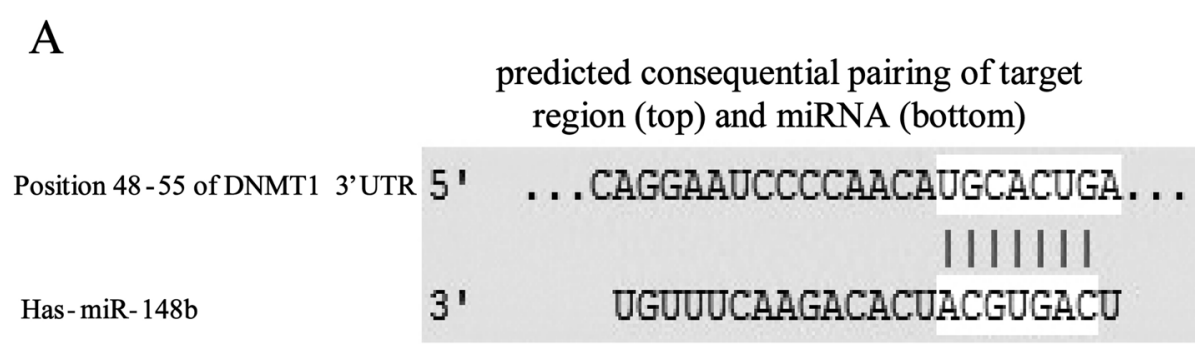

$\mathrm{B}$
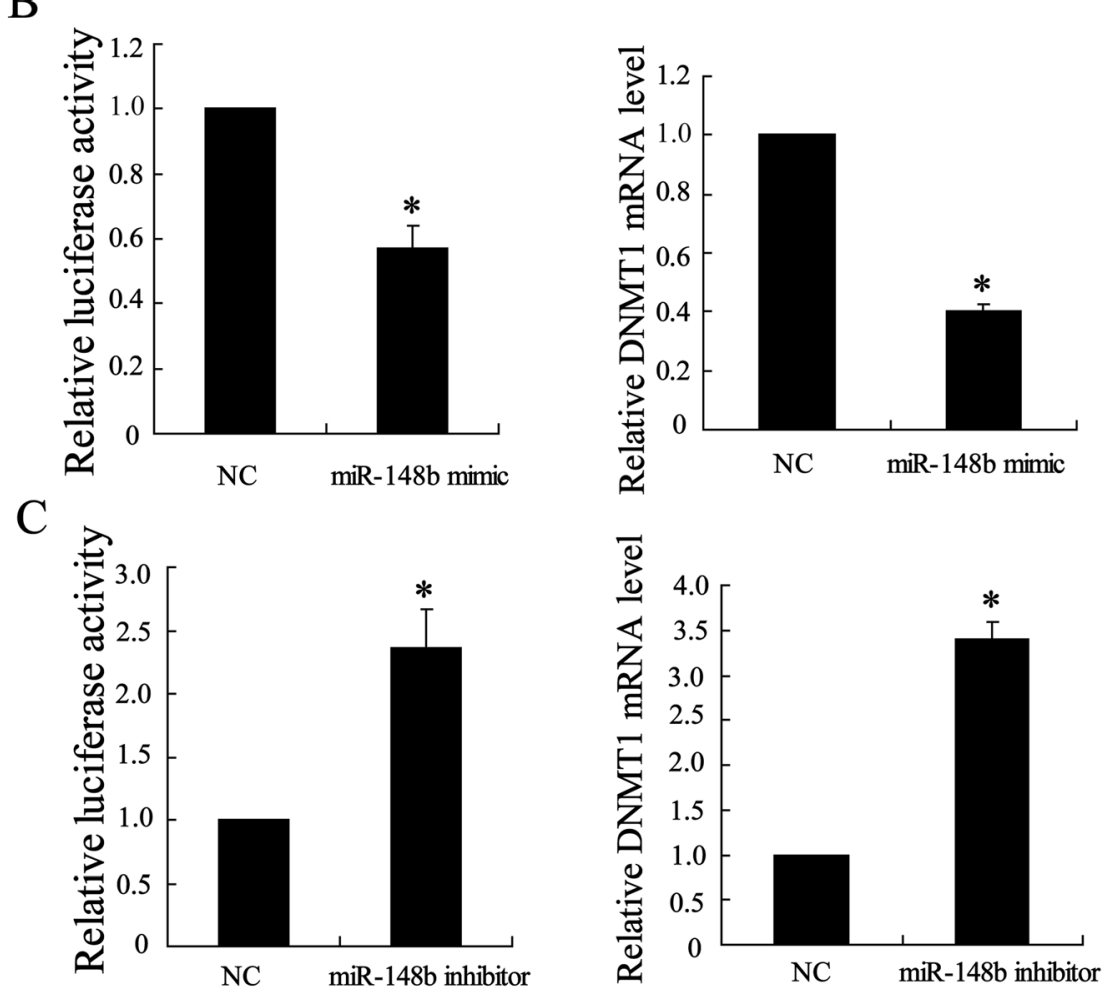

Figure 4 Targeting site of miR-148b in the DNMT1 3'UTR. (A) DNMT1 3'UTR was predicted a binding site for hsa-miR-148b. The mouse DNMT1 mRNA sequence is shown with potential binding sites indicated in white. The highly conserved mature miR-148b sequence in mammals and potential binding between the miR-148b seed region to the mouse DNMT1 3'UTR sequence are shown. (B) After A549/DDP transfected with miR-148b mimic for $24 \mathrm{~h}$, binding of miR-148b and DNMT1 was assessed by Luciferase reporter assays and expression of DNMT1 mRNA was assessed using Q-RT-PCR. (C) After A549 transfected with miR-148b ainhibitor for $24 \mathrm{~h}$, binding of miR-148b and DNMT1 was assessed by Luciferase reporter assays and expression of DNMT1 mRNA was assessed using Q-RT-PCR. Data were represented as mean \pm SD. *P $<0.05$ compared with NC

the data indicated a declining level of DNMT1 in A549/ DDP cell (Figure 5A) and SPC-A1/DPP cell (Figure 5C). To further investigate the effect of DNMT1 on cisplatinresistant cell chemoresistance, we analyzed the sensitivity of A549/DDP and SPC-A1/DPP cells to a series concentrations of ciaplatin after knock-down of DNMT1. The growth-inhibitory activities of ciaplatin in the A549/DDPDNMT1 and SPC-A1/DPP-DNMT1 were 1.2-fold lower than that in control cells $(P<0.05$, repectively; Figure 5B and $\mathrm{D})$. These demonstrate that down-regulation of DNMT1 could reverse the resistance of A549/DDP cells as well as SPC-A1/DPP to ciaplatin.

\section{Overexpression of DNMT1 reverses miR-148b-increased} cisplatin sensitivity of A549/DDP cells

To conform the regulating role of DNMT1 in miR-148b modulating the cisplatin sensitivity of the A549/DPP cells, the pcDNA3.1-DNMT1 or pcDNA3.1 negative control was transfected into the A549/DDP cells. Western blot assay showed an increased expression of DNMT1 in A549/DDP cells cotransfected with pcDNA3.1-DNMT1 and miR-148b mimics $(P<0.05$, Figure 6A). Next, cells were treated with a series concentration of ciaplatin to evaluate cells viability by MTT assay. As shown in Figure 6B, compared with miR-148b mimic NC, A549/DDP cells 

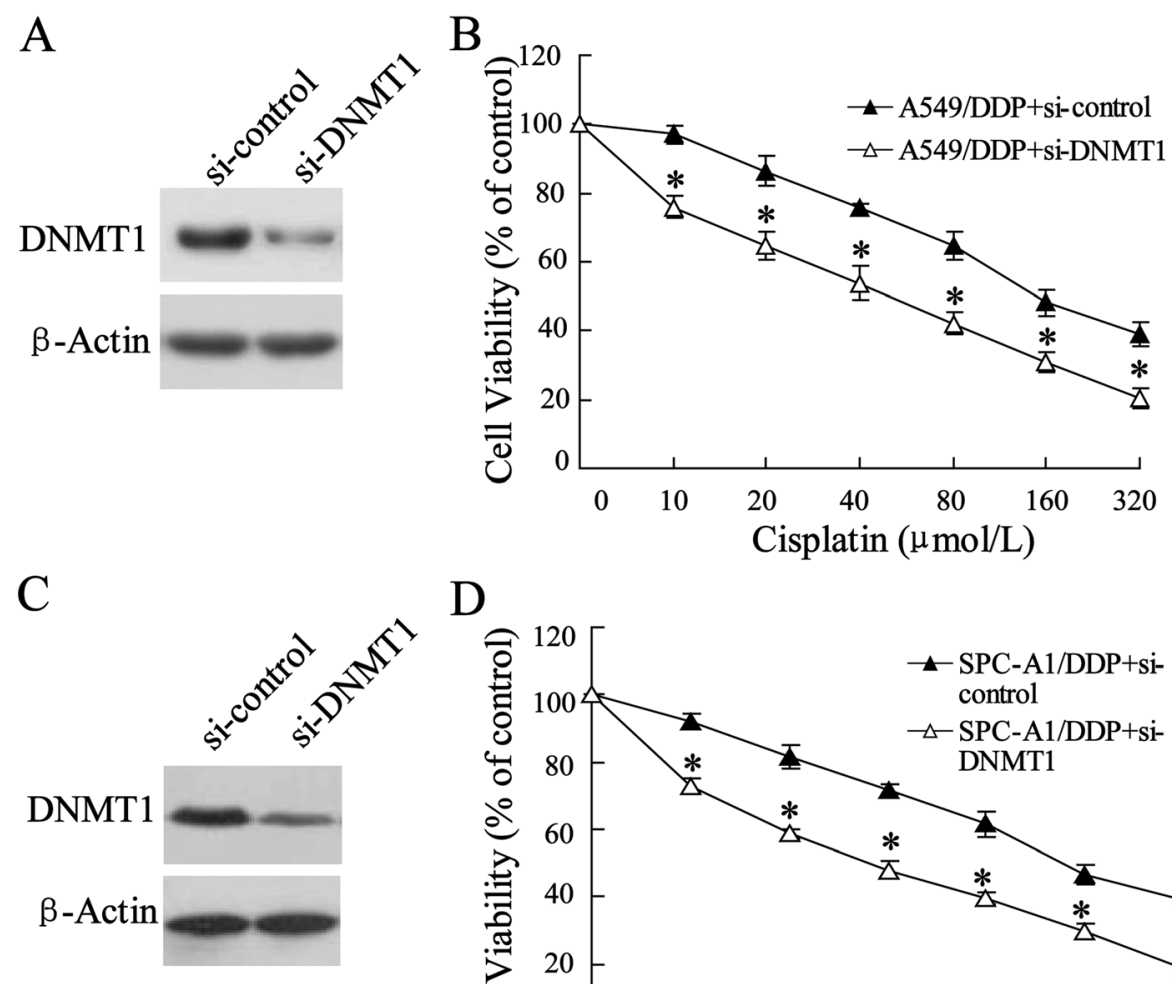

$\mathrm{D}$

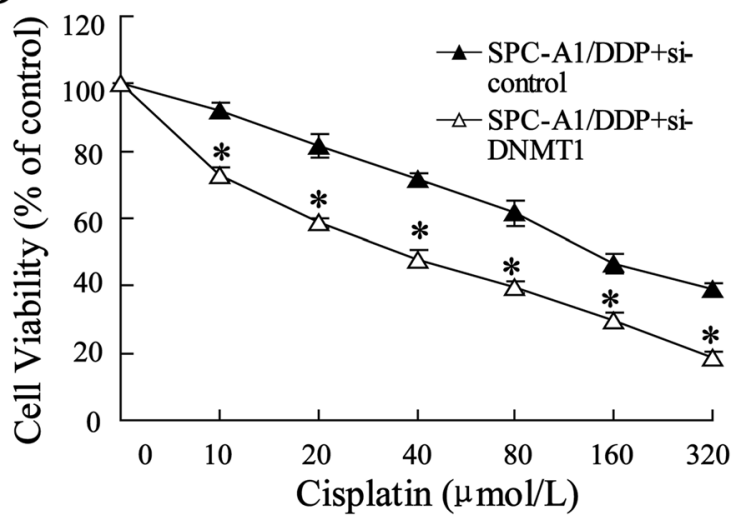

Figure 5 Effect of downregulated DNMT1 on cisplatin sensitivity of A549/DDP and SPC-A1/DDP cells. (A) A549/DDP cells were transfected with si-DNMT1. (B) 24 hours after incubation, the cell viability was assessed by MTT assay. (C) SPC-A1/DDP cells were also transfected with si-DNMT1 and (D) its cell viability was evaluated. Data were represented as mean \pm SD. ${ }^{*} P<0.05$ compared with cells treated with the same concentration of ciaplatin accordingly.

transfected with pcDNA3.1-DNMT1 reversed miR-148bincreased cisplatin sensitivity of A549/DDP cells $(P<0.05)$. Consistent with this data, apoptosis in A549/DDP cells transfected with pcDNA3.1-DNMT1 was lower than that in cells transfected with miR-148b mimic NC $(P<0.05$, Figure 6C).

\section{Discussion}

Cisplatin-based chemotherapy is the first-line chemotherapy for advanced-stage patients in various cancers at present. Despite a high rate of initial response, 5-year patient survival rate is very low resulting from tumors developing resistance to therapy [13]. Thus, an intense research underlying chemoresistance should be conducted to further establish better therapeutic approaches. In this research, we first detected the impact of miR-148b expression on ciaplatin resistance in NSCLC cells line and the results showed that miR-148b was notably downregulated in cisplatin-resistant NSCLC cell line (A549/ $\mathrm{DDP})$. The further analysis indicated that DNMT1 is the important target for miR-148b in the development of cisplatin resistance in NSCLC cells .

miR-148b is a member of miR-148/152 family, which has the mature structure of 21-22 nucleotides in length and the same seed sequence of approximately 6-7 nucleotides [14]. It has been demonstrated that down-regulated miR-148b is correlated with tumor formation, distant metastasis or worse prognosis in colorectal and gastric cancer $[7,8]$ and it also suppressed proliferation and migration of NSCLC cells [11] However, the study in relationship between miR-148b and chemotherapy resistance is very rare. Interestingly, as a member of miR-148/152 family, miR148a plays an important role in improving response to chemotherapy in sensitive and resistant cancers, such as oesophageal adenocarcinoma and squamous cell carcinoma cells [15] and hormone-refractory prostate cancer [16]. In this study, we focus on the changes in miR$148 \mathrm{~b}$ expression and hypothesized its involvement in chemotherapy resistance of NSCLC. Our data showed the down-regulated expression of miR-148b in A549/ DDP and SPC-A1/DPP cells compared with their parental 

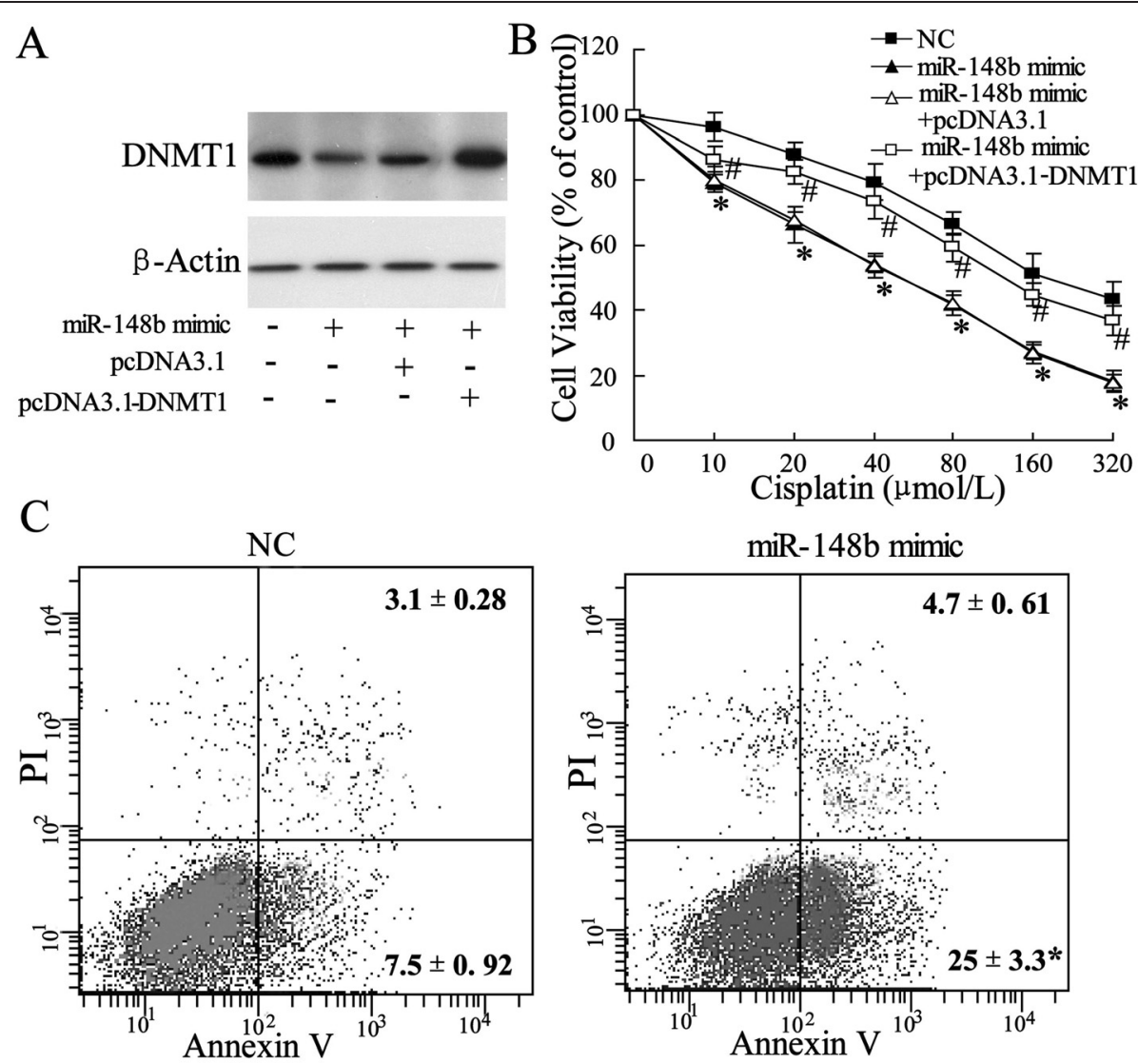

miR-148b mimic + pcDNA3.1

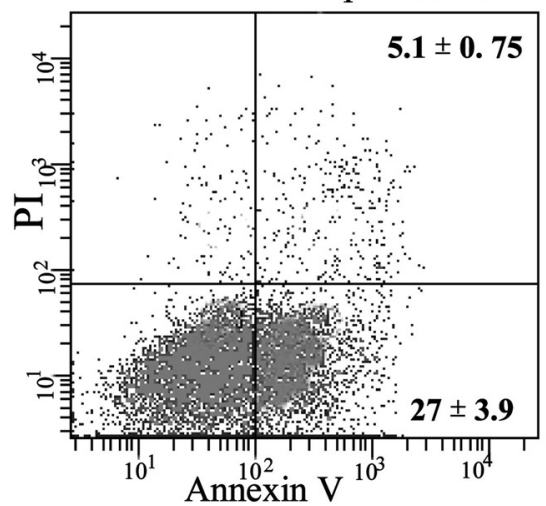

miR-148b mimic+pcDNA3.1-DNMT1

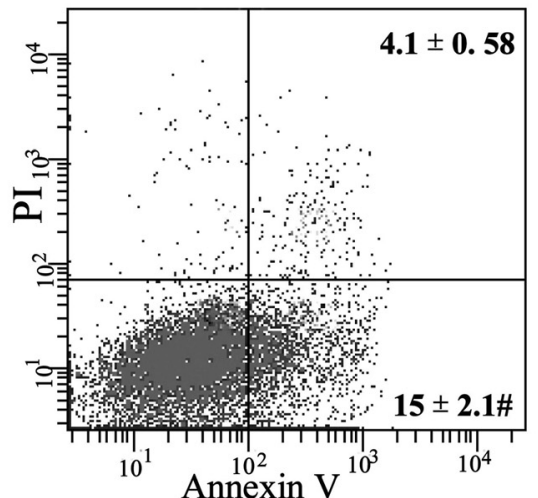

Figure 6 Effect of overexpressed DNMT1 on cisplatin sensitivity of A549/DDP cells. A549/DDP cells transfected with pcDNA3.1-DNMT1 or pcDNA3.1 were used in experiments. (A) Expression of DNMT1 was evaluated using western blot assay. (B) After transfected with miR-148b mimic for $24 \mathrm{~h}$, cells were incubated with various doses of cisplatin (10,20,40, 80, 160, 320 Mmol/L), and then cell viability was assessed using MTT assay. (C) After transfected with miR-148b mimic24 for $24 \mathrm{~h}$, cells were incubated with $10 \mu \mathrm{mil} / \mathrm{L}$ cisplatin, and then cell apoptosis was determined using flow cytometry. Data were represented as mean $\pm \mathrm{SD}$. ${ }^{*} P<0.05$ compared with NC; $\# \mathrm{P}<0.05$ compared with miR-148b mimic.

cell lines. Importantly, over-expression of miR-148b increased the sensitivity of A549/DDP as well as SPC-A1/ DPP cells to cisplatin measured by MTT and flow cytometry assay. Therefore, the results indicated that miR-148b could play a crucial role in the development of cisplatin resistance in NSCLC.
DNMTs is a kind of most abundant DNA methyltransferase in mammalian cells and mediate the transfer of methyl groups from S-adenosylmethionine to the 5 position of cytosine bases in the dinucleotide sequence $\mathrm{CpG}$ [17]. DNMTs play an important role in the epigenetic processes that including gene expression and the maintenance 
of genome integrity. Accumulated evidence has been demonstrated that DNMTs mediated transcriptional silencing in malignant tumors as well as in lung cancer. In this study, we also detected up-regulated DNMT1, DNMT3a and DNMT3b in A549/DDP cells. DNMTs regulation by miR$148 / 152$ family members has been reported in a number of human diseases $[18,19]$. However, the result in our research indicated that only DNMT1 was regulated by miR-148a. In addition, Xiang et al. reported that DNMT1 is a key target for miR-152 and miR-185 in ovarian cancer cisplatin resistance in vitro and in vivo [20]. DNMT1 was also involved in gastric carcinomas treated by neoadjuvant chemotherapy [21]. These results suggest that DNMT1 is an key target gene for miRNAs in development of chemotherapy resistance. Consistent with this, the result of TargetScan Release in our research indicated that DNMT1 acted as a potential target gene for miR-148b in its mRNA 3'UTR. Further protein expression assay showed that DNMT1 was up-regulated in A549/DDP, importantly, its expression was regulated by over-expression or downregulation of miR-148b in A549/DDP or A549.

\section{Conclusions}

In summary, this study has shown that the development of drug-resistant NSCLC cells is associated with the deregulation of miR-148b expression. Additionally, we have shown that expression of miR-148b is contradictory relationship with DNMT1 expression in the A549/DDP cells. Furthermore, the silence of DNMT1 expression in the A549/DDP cells reversed miR-148b-induced increases sensitivity of the drug-resistant cancer cells to cisplatin. Targeting the expressed miR-148b or its regulated gene may offer novel therapeutic approaches for the treatment of NSCLC.

\section{Competing interests}

The authors declare that they have no competing interests.

\section{Authors' contributions}

FM carried out the molecular genetic studies and drafted the manuscript. YL carried out the MTT assay and cell culture. YJ participated in the design of the study and performed the statistical analysis. CS conceived of the study, and participated in its design and coordination and helped to draft the manuscript. All authors read and approved the final manuscript.

\section{Acknowledgement}

Chengguang Sui contributed towards the study by making substantial contributions to conception, design, acquisition of data, or analysis and interpretation of data. It is also very grateful for all authors make great efforts.

Received: 26 November 2014 Accepted: 14 April 2015

Published online: 28 April 2015

\section{References}

1. Chen Y, Peng W, Lu Y, Chen J, Zhu YY, Xi T. MiR-200a enhances the migrations of A549 and SK-MES-1 cells by regulating the expression of TSPAN1. J Biosci. 2013;38(3):523-32.

2. Calbo J, Meuwissen R, van Montfort E, van Tellingen O, Berns A. Genotypephenotype relationships in a mouse model for human small-cell lung cancer. Cold Spring Harb Symp Quant Biol. 2005;70:225-32.
3. Verdecchia A, Francisci S, Brenner H, Gatta G, Micheli A, Mangone L, et al. Recent cancer survival in Europe: a 2000-02 period analysis of EUROCARE-4 data. Lancet Oncol. 2007;8(9):784-96.

4. Bartel DP. MicroRNAs: genomics, biogenesis, mechanism, and function. Cell. 2004;116(2):281-97.

5. Chitwood DH, Timmermans MC. Small RNAs are on the move. Nature. 2010;467(7314):415-9.

6. Zhao G, Zhang J-G, Liu Y, Qin Q, Wang B, Tian K, et al. MiR-148b functions as a tumor suppressor in pancreatic cancer by targeting AMPKa1. Mol Cancer Ther. 2013;12(1):83-93.

7. Azizi M, Teimoori-Toolabi L, Arzanani MK, Azadmanesh K, Fard-Esfahani P, Zeinali S. MicroRNA-148b and microRNA-152 reactivate tumor suppressor genes through suppression of DNA methyltransferase-1 gene in pancreatic cancer cell lines. Cancer Biol Ther. 2014;15(4):0-1.

8. Wang G, Cao X, Lai S, Luo X, Feng Y, Wu J, et al. Altered p53 regulation of miR-148b and p55PIK contributes to tumor progression in colorectal cancer. Oncogene. 2014;34(7):912-21.

9. Song YX, Yue ZY, Wang ZN, Xu YY, Luo Y, Xu HM, et al. MicroRNA-148b is frequently down-regulated in gastric cancer and acts as a tumor suppressor by inhibiting cell proliferation. Mol Cancer. 2011;10(1):1.

10. Sandhu R, Rivenbark AG, Mackler RM, Livasy CA, Coleman WB. Dysregulation of microRNA expression drives aberrant DNA hypermethylation in basal-like breast cancer. Int J Oncol. 2014;44(2):563-72.

11. Liu GL, Liu X, Lv XB, Wang XP, Fang XS, Sang Y. miR-148b functions as a tumor suppressor in non-small cell lung cancer by targeting carcinoembryonic antigen (CEA). Int J Clin Exp Med. 2014;7((8):1990.

12. Tang M1, Xu W, Wang Q, Xiao W, Xu R. Potential of DNMT and its epigenetic regulation for lung cancer therapy. Curr Genomics. 2009;10(5):336-52.

13. Wozniak AJ, Gadgeel SM. Adjuvant therapy for resected non-small cell lung cancer. Ther Adv Med Oncol. 2009;1(2):109-18.

14. Chen Y, Song YX, Wang ZN. The microRNA-148/152 family: multi-faceted players. Mol Cancer. 2013;12:3.

15. Hummel R, Watson DI, Smith C, Kist J, Michael MZ, Haier J, et al. Mir-148a improves response to chemotherapy in sensitive and resistant oesophageal adenocarcinoma and squamous cell carcinoma cells. J Gastrointest Surg. 2011;15(3):429-38.

16. Fujita Y, Kojima K, Ohhashi R, Hamada N, Nozawa Y, Kitamoto A, et al. MiR-148a attenuates paclitaxel resistance of hormone-refractory, drug-resistant prostate cancer PC3 cells by regulating MSK1 expression. J Biol Chem. 2010;285(25):19076-84.

17. Herman JG, Baylin SB. Gene silencing in cancer in association with promoter hypermethylation. N Engl J Med. 2003;349(21):2042-54.

18. Robertson KD, Uzvolgyi E, Liang G, Talmadge C, Sumegi J, Gonzales FA, et al. The human DNA methyltransferases (DNMTs) 1, 3a and 3b: coordinate mRNA expression in normal tissues and overexpression in tumors. Nucleic Acids Res. 1999;27(11):2291-8.

19. Tennis MA, VanScoyk MM, Wilson LA, Kelley N, Winn RA. Methylation of Wnt7a is modulated by DNMT1 and cigarette smoke condensate in non-small cell lung cancer. PLoS One. 2012;7(3), e32921.

20. Xiang Y, Ma N, Wang D, Zhang Y, Zhou J, Wu G, et al. MiR-152 and miR-185 co-contribute to ovarian cancer cells cisplatin sensitivity by targeting DNMT1 directly: a novel epigenetic therapy independent of decitabine. Oncogene. 2013;33(3):378-86.

21. Mutze K, Langer R, Schumacher F, Becker K, Ott K, Novotny A, et al. DNA methyltransferase 1 as a predictive biomarker and potential therapeutic target for chemotherapy in gastric cancer. Eur J Cancer. 2011;47(12):1817-25. 